\title{
The carotenoid-labeling method: measuring specific rates of carotenoid synthesis in natural phytoplankton communities
}

\author{
Ralf Goericke* , Nicholas A. Welschmeyer** \\ The Biological Laboratories, Harvard University, Cambridge, Massachusetts 01248, USA
}

\begin{abstract}
The physiological basis of the carotenoid- $-{ }^{14} \mathrm{C}$-labeling method for the determination of growth rates $\left(\mu, \mathrm{d}^{-1}\right)$ of specific groups of microalgae was established in the laboratory and the method was tested in the subarctic Pacific and in Chesapeake Bay (USA). ${ }^{14} \mathrm{C}$-labeling patterns of carotenoids in a variety of algal species grown in batch cultures were described successfully with a simple precursor-pigment model whose free parameters, the specific rate of carotenoid synthesis $\left(\mu_{\text {caro, }} \mathrm{d}^{-1}\right)$ and the precursor and the pigment turnover rates $\left(\mathrm{d}^{-1}\right)$, were determined by least squares analysis. All xanthophylls except peridinin had turnover rates that did not differ significantly from zero; the turnover rate of peridinin was $0.7 \mu$. Precursor turnover rates varied from about $5 \mu$ for fucoxanthin to $36 \mu$ for lutein. We propose to use the precursor-pigment model to calculate $\mu_{\text {caro }}$ from the amount of ${ }^{14} \mathrm{C}$ incorporated into carotenoids and values of carotenoid precursor turnover rates, which are assumed to be known a priori. A well-constrained estimate of the fucoxanthin precursor turnover rate is presented here. It was shown for laboratory cultures that the carotenoid-labeling method is capable of measuring specific rates of carotenoid synthesis and that these rates equal rates of cell growth only when growth is balanced. We demonstrated that pigment synthesis and carbon fixation can be unbalanced in natural phytoplankton populations due to the effects of light perturbations and growth under a natural photocycle. We recommend that labeling experiments last $24 \mathrm{~h}$ to average rates of synthesis over the diel photoperiod since rates of carotenoid synthesis and cell growth can be unbalanced at any time during the photoperiod. The field experiments also demonstrated that the carotenoid-labeling method is a powerful tool to study the physiological ecology of natural populations of phytoplankton.
\end{abstract}

\section{INTRODUCTION}

Properties of marine plankton communities such as biomass distributions, primary production, and new production depend among other factors on the structure of the phytoplankton community. Phytoplankton communities usually consist of a large number of species, each interacting in a unique way with the biotic and abiotic environment. It is often impossible to study the individual species in the field because of the diffi-

Present addresses:

- Department of Chemistry, Woods Hole Oceanographic Institution, Woods Hole, Massachusetts 02543, USA

- Moss Landing Marine Laboratories, Moss Landing, California 95039-0450, USA, and Monterey Bay Aquarium Research Institute, Pacific Grove, California 93950, USA culty to physically separate these for analysis. Instead, algal assemblages placed above the species level, or at ataxonomic levels, based on size, shape or chemical characteristics, are studied. Pigments, in particular carotenoids, have been used as chemotaxonomic markers to differentiate and estimate the biomass of different components of phytoplankton communities (Jeffrey 1980). This approach is taxonomic, since the presence of particular xanthophylls is usually restricted to specific taxa of algae (Goodwin \& Britton 1988).

Rates of growth $(\mu)$ and loss of the individual species determine the structure of phytoplankton communities. Measuring these rates and understanding their controls is one of the fundamental goals of phytoplankton ecology. Methods to determine rates of growth for specific groups of microalgae range from 
measuring the frequency of dividing cells (McDuff \& Chisholm 1982) to autoradiography (Douglas 1984). However, none of these methods group the whole phytoplankton community into assemblages for which physiological properties can be measured. Gieskes \& Kraay (1989) proposed the calculation of growth rates of individual groups of algae from the amount of ${ }^{14} \mathrm{C}$ incorporated into taxon-specific carotenoids, in strict analogy with the chlorophyll-labeling method of Redalje \& Laws (1981). Gieskes \& Kraay assumed that the ${ }^{14} \mathrm{C}$-specific activity of total cell carbon and carotenoids are equal after relatively short incubation times.

However, we have recently shown that this assumption is not necessarily true for chlorophyll $a$ (chl a) and fucoxanthin in the diatom Thalassiosira weissflogii grown in dilute batch cultures (Goericke \& Welschmeyer $1992 \mathrm{a}, \mathrm{b})$. These experiments with $T$. weissflogi demonstrated that the labeling kinetics of fucoxanthin and the 2 chl a subunits, porphyrin and phytol, are best described by equations corresponding to a simple precursor $(F)$ - pigment (P) model, the F-P model (Goericke \& Welschmeyer 1992a), rather than by the equations given by Redalje \& Laws (1981). The equation corresponding to the F-P model is:

$$
\cdot P=1-\frac{b}{b-a} \exp (-a t)-\frac{a}{a-b} \exp (-b t),
$$

where $\cdot P=$ relative ${ }^{14} \mathrm{C}$-specific activity of a pigment $\left[\left(\mathrm{dpm} \mu \mathrm{g}^{-1} \mathrm{C}_{\mathrm{p}}\right) /\left(\mathrm{dpm} \mu \mathrm{g}^{-1} \mathrm{C}_{\mathrm{DIC}}\right)\right.$, where DIC $=$ dissolved inorganic carbon]; $t=$ incubation time; $a=$ $k_{\mathrm{P}}+\mu_{\mathrm{P} i} b=k_{\mathrm{F}}+\mu_{\mathrm{P} i} k_{\mathrm{P}}=$ turnover rate of $\mathrm{P}$; $k_{\mathrm{F}}=$ turnover rate of $\mathrm{F}_{\text {; }}$ and $\mu_{\mathrm{P}}=$ specific rate of pigment synthesis. Units of all rates are $\mathrm{d}^{-1}$.

We estimated the parameters $a$ and $b$ from the time course of $" P$ by fitting Eq. (1) to measured values of $\cdot P$ and calculated the turnover rates of $\mathrm{chl} a$ or fucoxanthin and the turnover rates of their immediate precursors by subtracting $\mu_{\mathrm{p}}$ from the estimated values of $a$ and $b$ (Goericke \& Welschmeyer 1992a, b). $\mu_{\mathrm{P}}$ was calculated from pigment concentration changes in the cultures. Turnover rates of chl $a$ and fucoxanthin in Thalassiosira weissflogii were not significantly different from zero. The precursor turnover rates of chl $a$ and fucoxanthin scaled as constant multiples of the growth rate, with average values of $31 \mu$ for chl $a$ and $5 \mu$ for fucoxanthin. These values imply that the immediate precursors of chl a and fucoxanthin require an amount of time equivalent to 0.2 to 0.5 cell doublings to attain approximate isotopic equilibrium with the pool of dissolved inorganic carbon

Chlorophyll $a-{ }^{14} \mathrm{C}$-labeling experiments with a variety of other algal species (Goericke \& Welschmeyer 1993) also demonstrated that rates of chl a turnover were generally zero, while turnover rates of the pre- cursor of chl a were constant multiples of the specific rate of chl a synthesis. Based on these results we proposed the calculation of $\mathrm{chl}$ a synthesis rates from an equation similar to Eq. (1) for values of ${ }^{*} P_{\mathrm{ch} \text { a }}$ measured in the field. We also showed that ${ }^{14} \mathrm{C}$-labeling rates of chl a scale with specific rates of chl a synthesis rather than rates of ${ }^{14} \mathrm{C}$-fixation in photoadapting phytoplankton (Goericke \& Welschmeyer 1993). Thus, rates of chl a synthesis correspond to rates of growth only if growth is balanced, i.e. 'when the amounts of all cellular components increase exponentially at the same rate' (Shuter 1979).

Here we present results of laboratory carotenoidlabeling experiments with a variety of algal species to corroborate our previous results for fucoxanthin in Thalassiosira weissflogii (Goericke \& Welschmeyer $1992 \mathrm{~b}$ ). Based on these results we propose an improved method for the measurement of specific rates of carotenoid synthesis from rates of ${ }^{14} \mathrm{C}$-incorporation. Rates of carotenoid synthesis will be calculated by solving Eq. (1) for $\mu$ with values of ${ }^{\circ} P_{\text {carotenoid }}$ and $t$ measured in the field. We will show for natural populations of microalgae that ${ }^{14} \mathrm{C}$-labeling patterns of carotenoids are well described by the F-P model. We also studied the effect of photoadaptation and the effect of the natural light cycle on the ${ }^{14} \mathrm{C}$-labeling rates of carotenoids to determine if carotenoid-labelingmethod-based estimates of growth or pigment synthesis are biased under those conditions

\section{METHODS}

Culture experiments. Pigment-labeling experiments were performed with algae growing under steady state conditions or under induced conditions of photoadaptation. Steady state experiments were performed in dilute batch cultures under continuous light. Growth in such cultures is balanced (cf. Shuter 1979) as long as cultures are dilute and nutrients are not depleted. We chose continuous light instead of light: dark cycles to ensure that rates of carbon fixation and pigment synthesis were equal at all times. A light-shift experiment was performed with 3 cultures of Thalassiosira weissflogii which had grown at an irradiance of $125 \mu$ Einst $\mathrm{m}^{-2} \mathrm{~s}^{-1}$ for 4 generations. At the beginning of the experiment, after cultures had been inoculated with ${ }^{14} \mathrm{C}$ bicarbonate, one culture was shifted to $47 \mu$ Einst $\mathrm{m}^{-2}$ $\mathrm{s}^{-1}$, a second to $288 \mu$ Einst $\mathrm{m}^{-2} \mathrm{~s}^{-1}$, and a third was kept at $125 \mu$ Einst $\mathrm{m}^{-2} \mathrm{~s}^{-1}$. Details of the culturing, sampling, and sample preparation methods as well as the highpressure liquid chromatography (HPLC) system used, were described in Goenicke \& Welschmeyer (1993). The particular methods used to purify carotenoids are described below. A variety of labeling experiments 
were performed in the subarctic Pacific and in Chesapeake Bay (USA) to study patterns of carotenoid ${ }^{14} \mathrm{C}$-labeling and synthesis in the field. The sampling and experimental protocols used in the field were described in Goericke \& Welschmeyer (1993).

Chromatography. Analytical and preparative work on raw pigment extracts was carried out by reversephase HPLC. Peridinin, 19'-hexanoyloxy-fucoxanthin (19'-hex-fucox), and fucoxanthin were isolated from acetone extracts of algal cultures as described previously for fucoxanthin (Goericke \& Welschmeyer $1992 b)$. Preparations of these pigments were shown to be radiochemically pure (see below). Lutein, zeaxanthin and $\beta$-carotene isolated from extracts of algal cultures as described above were contaminated by colorless compounds, as evidenced by specific activities (dpm $\mu \mathrm{g}^{-1} \mathrm{C}$ ) of the raw pigment preparations that were higher than the specific activity of the inorganic carbon. To obtain radiochemically pure pigment preparations the pigments were saponified and rechromatographed as described previously for diadinoxanthin (Goericke \& Welschmeyer 1992b).

HPLC solvent eluting immediately before and after pigment fractions of interest was monitored for ${ }^{14} \mathrm{C} \mathrm{ac}$ tivity throughout the development of chromatographic procedures. If ${ }^{14} \mathrm{C}$ is detected in such fractions, it indicates that the pigment may be contaminated by compounds that do not absorb light in the visible range (Goericke 1992). Using this monitoring method, we observed high levels of contamination in peridinin, $19^{\prime}$-hex-fucox, and fucoxanthin isolated from field samples. We achieved radiochemical purity of these pigments using the following method. Pigment frac- tions were concentrated individually on Baker C-18 sample preparations columns. The columns were dried by forcing nitrogen through the columns for $5 \mathrm{~min}$ and the pigments were eluted with $0.5 \mathrm{ml} 100 \%$ acetone. The acetone was evaporated under a stream of nitrogen and the pigment was taken up with $40 \mu \mathrm{l} 100 \%$ acetone to which $360 \mu$ l hexane were added. For final purification each pigment was chromatographed individually on a normal phase HPLC system (Fiksdahl et al. 1978), using a $30 \mathrm{~cm}$ Rainin Microsorb $5 \mu \mathrm{m}$ silica column and the following gradients (time (min); $\%$ hexane, \% acetone for: peridinin, fucoxanthin and $19^{\prime}$-hex-fucox $=[\{0 ; 90,10\},\{3 ; 80,20\},\{5 ; 70,30\},\{7 ; 60$, $40\},\{10 ; 60,40\}] ;$ zeaxanthin and alloxanthin $=[\{0 ; 80$, $20\},\{2 ; 70,30\},\{5 ; 60,40\},\{8 ; 60,40\}]$.

The chromatographic procedures were tested by isolating pigments from homogeneously labeled algae grown in seawater of known ${ }^{14} \mathrm{C}$-activity. Relative specific activities of radiochemically pure pigments isolated from such cultures are expected to equal 1.0 or less if the algae fractionate against the heavier isotope ${ }^{14} \mathrm{C}$ (Goericke \& Welschmeyer 1992a). The relative specific activities of all carotenoids were less than 1.0 (Table 1), which indicates that carotenoids isolated using the above methods were radiochemically pure. The relative specific activities were on the average 0.92 , suggesting an $8 \%$ isotopic discrimination against the heavier isotope ${ }^{14} \mathrm{C}$. Carotenoid specific activities were divided by 0.92 to correct for the isotopic discrimination against ${ }^{14} \mathrm{C}$.

The extinction coefficients used for quantifying the pigments are listed in Table 1 . We determined the coefficients for $19^{\prime}$-hex-fucox and alloxanthin using the

Table 1. Some physical characteristics and relative specific activities for a variety of carotenoids isolated from homogeneously labeled algae. MW: molecular weight; $\alpha(\lambda)$ : extinction coefficient of the pigment $\left(\mathrm{l} \mathrm{g}^{-1} \mathrm{~cm}^{-1}\right.$ ) dissolved in acetone (but lutein in ethanol) at the indicated wavelength $(\lambda)_{;} \lambda_{\text {max }}$ : wavelength maximum determined in this study; $\cdot P_{\text {carotenoid }}$ : relative specific activities of the carotenoid isolated from homogeneously labeled cultures; $n$ : number of replicate cultures on which the value of - $P_{\text {carotenor }}$ is based ( $P$ was determined for each culture 2 to 7 times); $k_{\mathrm{F}}$ : the value of the growth-rate-normalized pigment precursor turnover rate that was used in this study to calculate specific rates of pigment synthesis for field experiments

\begin{tabular}{|c|c|c|c|c|c|c|c|c|}
\hline Pigment & Formula & MW & $\alpha(\lambda)$ & Source $^{a}$ & $\lambda_{\max }$ & - $P_{\text {carotenoid }}$ & $\mathrm{n}$ & $k_{\mathrm{F}}$ \\
\hline Zeaxanthin & $\mathrm{C}_{40} \mathrm{H}_{56} \mathrm{O}_{2}$ & 568 & $234 \quad(452)$ & (1) & 454 & 0.87 & 2 & 16 \\
\hline Lutein & $\mathrm{C}_{40} \mathrm{H}_{56} \mathrm{O}_{2}$ & 568 & $255(445)$ & (1) & 448 & 0.97 & 2 & 36 \\
\hline Peridinin & $\mathrm{C}_{39} \mathrm{H}_{50} \mathrm{O}_{7}$ & 630 & $134(466)$ & (2) & 470 & 0.91 & 4 & 15 \\
\hline Fucoxanthin & $\mathrm{C}_{42} \mathrm{H}_{58} \mathrm{O}_{6}$ & 658 & 160 (449) & (2) & 449 & 0.92 & 9 & 5 \\
\hline $19^{\prime}$-hex-fucox & $\mathrm{C}_{48} \mathrm{H}_{68} \mathrm{O}_{8}$ & 772 & 151 (449) & $(3,5)$ & 448 & $--^{c}$ & 3 & 5 \\
\hline $19^{\prime}$-but-fucox & $\mathrm{C}_{46} \mathrm{H}_{64} \mathrm{O}_{8}$ & 744 & $145^{b}(449)$ & (3) & 448 & - & - & 5 \\
\hline Alloxanthin & $\mathrm{C}_{40} \mathrm{H}_{52} \mathrm{O}_{2}$ & 564 & $227(454)$ & $(4,5)$ & 454 & $-{ }^{c}$ & 3 & 20 \\
\hline \multicolumn{9}{|c|}{$\begin{array}{l}\text { "References for the absorption coefficient and/or the formula: } \\
\text { (1) Davies (1976); (2) Jensen (1978); (3) Liaaen-Jensen (1985); (4) Pennington et al. (1985); (5) this study }\end{array}$} \\
\hline \multicolumn{9}{|c|}{$\begin{array}{l}{ }^{6} \text { The specific extinction coefficient of } 19^{\prime} \text {-butanoyloxy-fucoxanthin was calculated assuming that its molar extinction } \\
\text { coefficient equals the molar extinction coefficient of } 19^{\prime} \text {-hexanoyloxy-fucoxanthin }\end{array}$} \\
\hline
\end{tabular}


method of Roberts (1969). Chl $a$ and 19'-hex-fucox or alloxanthin were isolated from homogeneously labeled cultures of Emiliania huxleyi or Chroomonas salinas. The pigments were purified as described above and transferred to acetone for spectrophotometric measurements. ${ }^{14} \mathrm{C}$-activities of the pigment solutions (dpm $\mathrm{ml}^{-1}$ ) were determined by scintillation counting. Carotenoid concentrations were calculated from the solutions' ${ }^{14} \mathrm{C}$-activity assuming that carotenoids and chl a isolated from homogeneously labeled cultures have identical specific activities. This assumption was shown to be true for other algae, for which specific activities of chl a and a major xanthophyll were determined independently (Goericke \& Welschmeyer 1992a). The extinction coefficients for $19^{\prime}$-hex-fucox and alloxanthin (Table 1) were calculated from the ratio of the concentration and the absorbance.

Data analysis. For cultures $\mu_{\mathrm{p}}$ was determined by fitting an exponential to the time course of the pigment concentrations. The parameters of the F-P model, $a$ and $b$, were determined for all carotenoids, except $\beta$-carotene, by fitting Eq. (1) to the time course of $P$ using the nonlinear curve fitting program SYSTAT. Pigment turnover rates, $k_{\mathrm{P}}$ and pigment precursor turnover rates, $k_{\mathrm{F}}$, were calculated by subtracting $\mu_{\mathrm{P}}$ from $a$ and $b$. In the case of $\beta$-carotene we fitted equations corresponding to a Precursor-(Pigment 1 , Pigment 2) model, the $F-\left(P_{1}, P_{2}\right)$ model, and a Precursor-Pigment 1-Pigment 2 model, the F- $P_{1}-P_{2}$ model (see Fig. 1B \& C, respectively, of Goericke \& Welschmeyer 1992a) to the time course of ${ }^{*} P_{\beta \text {-carotene. }}$ Explained variance usually ranged from 99.0 to $99.9 \%$. The goodness of fit was judged by a random distribution of residuals. It was not possible to estimate $95 \%$ confidence intervals for individual parameter estimates reliably. However, for the parameters of the F-P model these can be estimated from the parameter estimates of replicate experiments (Goericke \& Welschmeyer 1992a, b). Standard deviations for estimates of Eq. (1) parameters are 0.05 for values of $k_{p}$ in the range -0.15 to 0.15 . The coefficient of variation for corresponding estimates of $k_{F}$ was ca $30 \%$. We will use these values subsequently for hypothesis testing. All turnover rates $(k)$ were converted to growth-rate-normalized rates (i.e. GRN-turnover rates) to facilitate the comparison of rates for algae, growing at differing rates. GRN-rates are dimensionless and represent those rates that would have been mea- sured had the algae grown at a rate of $1.0 \mathrm{~d}^{-1}$. We determined specific rates of pigment synthesis from labeling experiments conducted in the field by solving Eq. (1) for $\mu_{p}$ for measured values of $\cdot P$ and incubation time $t$. Eq. (1) was solved for $\mu_{\mathrm{p}}$ numerically with the secant method (Maron 1982). Note however, that numerical solutions for Eq. (1) can also be found using spreadsheet functions such as the Microsoft Excel function 'Goal Seek'. To calculate $\mu$ we used those values of $k_{\mathrm{F}}$ that are listed in Table 1 and assumed that $k_{\mathrm{p}}$ is zero (see below).

\section{RESULTS}

\section{Laboratory studies}

The time course of ${ }^{14} \mathrm{C}$-incorporation into carotenoids in 5 different species of algae was analyzed (Table 2). An experiment with Synechococcus bacillaris is shown in detail in Fig. 1. At time zero the culture was inoculated with $\mathrm{H}^{14} \mathrm{CO}_{3}^{-}$and sampled repeatedly for the determination of pigment concentrations and pigment specific activity until growth declined. Rates of cell growth, or rather specific rates of pigment concentration change, were determined by fitting an exponential (solid lines in Fig. 1A) to the time course of pigment concentrations. The relative specific activity $(\cdot P)$ of zeaxanthin and $\beta$-carotene increased in proportion to growth (Fig. 1B). The specific activity of $\beta$ carotene increased somewhat faster than the specific activity of zeaxanthin. Transformed relative specific activities $(\cdot R)$ were obtained from ${ }^{*} R=\ln \left(1-{ }^{*} P\right)$ and

Table 2. Results of the 6 carotenoid-labeling experiments reported here and a summary of previously reported data. The parameters of Eq. (1), $k_{\mathrm{P}}$ and $k_{\mathrm{F}}$, were estimated by fitting labeling data to Eq. (1) using a nonlinear fitting procedure. GRN $-k_{\mathrm{p}}$ is the growth-rate-normalized pigment turnover rate. The probability that the rate is significantly different from zero is indicated by: ${ }^{\text {ns }}$ not significantly different; ${ }^{\prime}$ significantly different $(p<0.05)$. GRN- $k_{F}$ is the growth-rate-normalized pigment precursor turnover rate. GRN-turnover rates are dimensionless

\begin{tabular}{|c|c|c|c|}
\hline Species & Pigment & $G R N-k_{p}$ & GRN-k \\
\hline Phaeodactylum tricornutum & Fucoxanthin & $0.03^{\mathrm{ns}}$ & 3.8 \\
\hline Emiliania huxleyi & 19'-hex-fucox & $-0.03^{\mathrm{ns}}$ & 7.6 \\
\hline Synechococcus bacillaris & Zeaxanthin & $0.09^{n s}$ & 16.4 \\
\hline Dunaliella tertiolecta & Lutein & $0.09^{\mathrm{ns}}$ & 35.9 \\
\hline Amphidinium carterae ${ }^{a}$ & Peridinin & $0.77^{*} \cdot$ & 12.9 \\
\hline A. carterae & Peridinin & $0.60^{*}$ & 17.1 \\
\hline Thalassiosira weissflogii ${ }^{\mathrm{b}}$ & Fucoxanthin & $0.00^{\text {ns }}$ & 5.1 \\
\hline Species listed above ${ }^{c}$ & Chlorophyll a & $0.05^{\mathrm{ns}}$ & 15.0 \\
\hline \multicolumn{4}{|c|}{${ }^{a}$ These represent 2 independent experiments } \\
\hline \multicolumn{4}{|c|}{$\begin{array}{l}{ }^{\mathrm{b}} \text { These values are the mean parameter values for } 14 \text { expenments with } \\
T \text { weissflogi that were reported by Goericke \& Welschmeyer (1992b) }\end{array}$} \\
\hline \multicolumn{4}{|c|}{ 'Summarized from Goericke \& Welschmeyer (1993) } \\
\hline
\end{tabular}




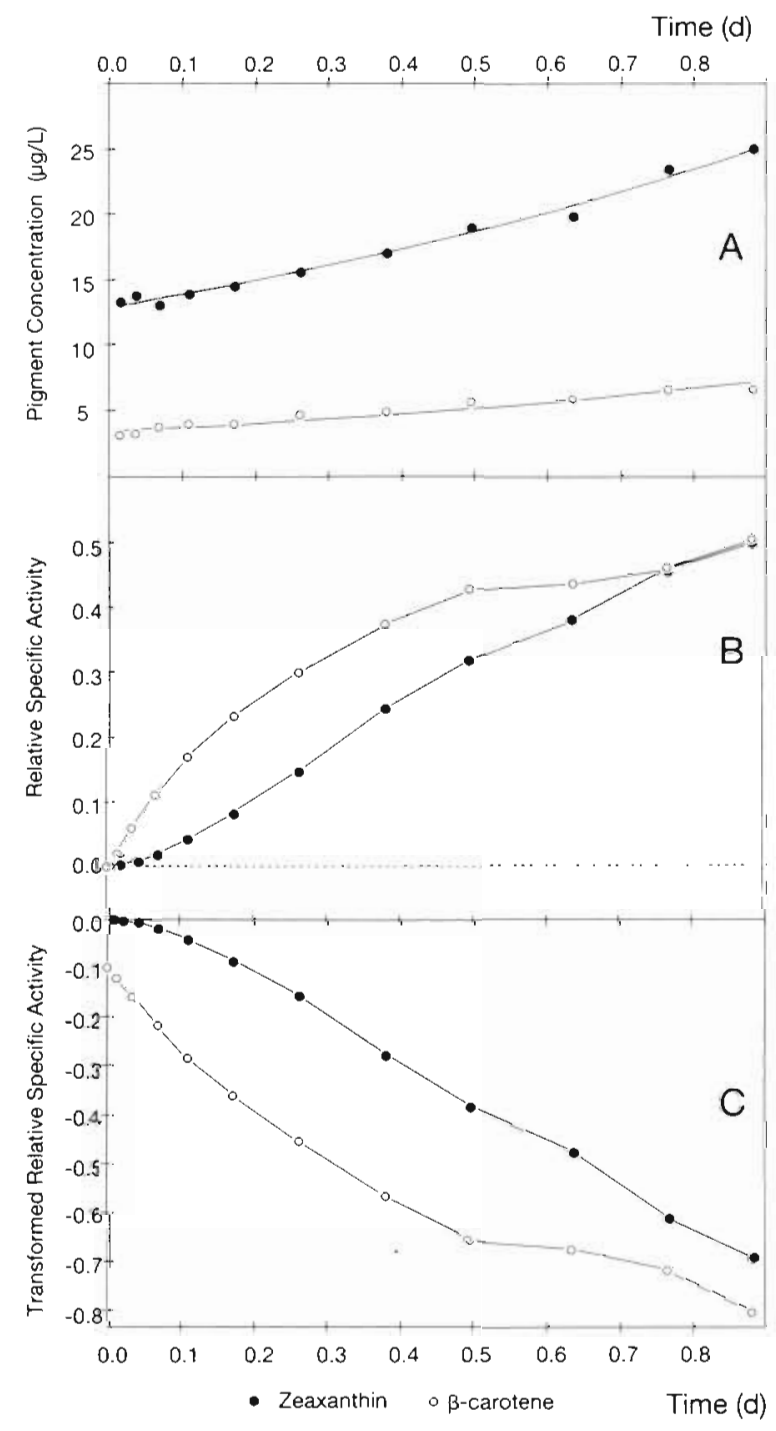

Fig. 1. A carotenoid-labeling experiment with Synechococcus bacillaris. Data are plotted as a function of time. (A) Concentrations of zeaxanthin and $\beta$-carotene $\left(\mu \mathrm{gl}^{-1}\right)$. Solid lines represent an exponential fit to the data. (B) Relative specific activity $(\cdot P)$ of zeaxanthin and $\beta$-carotene. (C) Transtormed relative specific activities, $\cdot R=\ln \left(1-^{-} P\right)$, of zeaxanthin and

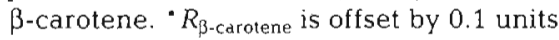

plotted against time (Fig. 1C), thus transforming the dominant signal in the labeling data due to growth alone, $\cdot P=1-\exp (-\mu t)$ (Goericke \& Welschmeyer 1992a), to a straight line with an intercept of zero and a slope which equals negative $\mu_{\mathrm{p}}$. Deviations of $\cdot R(t)$ from a straight line with a slope of negative $\mu_{\mathrm{p}}$ are related to the effects of pigment turnover, precursor pools, or multiple pigment pools (Goericke \& Welschmeyer 1992a).

The transformed labeling pattern of zeaxanthin displays the characteristics of a pigment which is present as a single pool in the alga (Goericke \& Welschmeyer 1992a). The time that its immediate metabolic precursor requires to attain approximate isotopic equilibrium with the pool of inorganic carbon is reflected in the initial curvature of the labeling time course (Fig. 1C). The labeling time course approaches a straight line as $t$ increases; the negative value of the slope of the line corresponds to the rate at which zeaxanthin is synthesized, i.e. $k_{\mathrm{P}}+\mu_{\mathrm{P}}$. We determined the turnover rate of zeaxanthin and its precursor by fitting Eq. (1) to the time course of * $P$. The GRN-turnover rates of zeaxanthin and its precursor were 0.09 and 16 , respectively.

The transformed relative specific activity of $\beta$ carotene increased continuously with time; the last 3 time points show some perturbation, possibly from slight irradiance changes due to self-shading within the culture. These data points were excluded from the subsequent analysis. The initial curvature of the labeling pattern of $\beta$-carotene is inconsistent with the F-P model. Instead, we fit the two 3-pool models of Goericke \& Welschmeyer (1992a) to these data. Both models consist of 1 precursor pool and 2 pigment pools; the free parameters are the specific rate of carotenoid synthesis, the turnover rate of the precursor, the turnover rates of pigment Pools 1 and 2, and the ratio of pigment Pool 1 and the sum of Pools $1+2$. A fit of the F- $\mathrm{P}_{1}-\mathrm{P}_{2}$ model to the labeling data gave GRN-turnover rates of 11 and -0.04 for $\beta$-carotene Pools 1 and 2 , respectively, a ratio of $\beta$-carotene Pool 1 to total $\beta$-carotene of 0.24 , and a GRN-turnover rate of the precursor of 371 . Corresponding parameter values for a fit of the F- $\left(\mathrm{P}_{1}, \mathrm{P}_{2}\right)$ model to the time course of - $P_{\beta \text {-carotene }}$ were $11,-0.01,0.17$, and 244 . The good correspondence between the parameter estimates based on the 2 different models suggests that these estimates are relatively insensitive to the structure of the particular model used. We did not study the labeling of $\beta$-carotene in other algae because concentrations of $\beta$-carotene were generally too low to determine - $P_{\beta-\text { carotene }}$ accurately.

The transformed labeling kinetics of the major xanthophylls in the algae studied here are shown in Fig. 2. We fitted the F-P model to the data to determine pigment and pigment precursor turnover rates. The turnover rates of zeaxanthin, lutein, fucoxanthin and 19 '-hex-fucox were not significantly different from zero (Table 2). The GRN-turnover rates of peridinin in the 2 experiments with Amphidinium carterae were 0.60 and 0.77 , values significantly different from zero (Table 2). The GRN-turnover rates of the precursors of fucoxanthin and 19'-hex-fucox were 7.6 and 3.8, values similar to the average GRN-turnover rate of the fucoxanthin precursor in Thalssiosira weissflogui (Table 2). The GRN-turnover rates of the precursors of the other xanthophylls ranged from 13 to 36 (Table 2). 


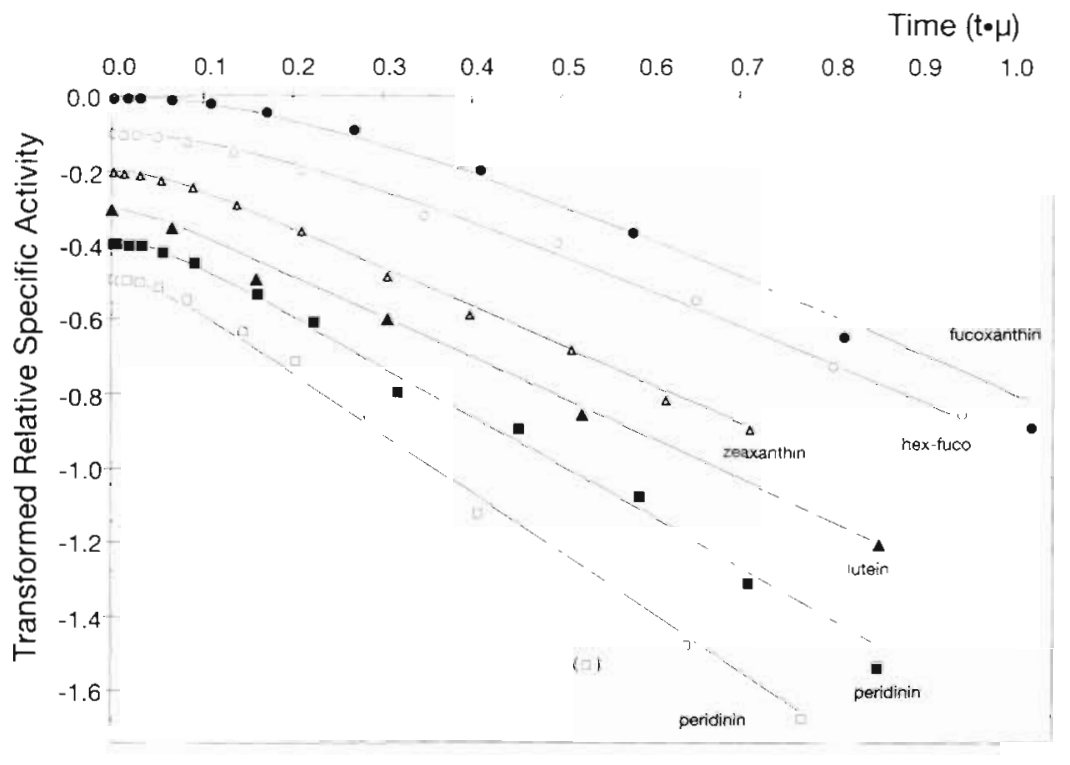

Fig. 2. Transformed relative specific activities ( $R$ ) of the major xanthophylls for labeling experiments with Phaeodactylum tricornutum (fucoxanthin). Amphidinium carterae (peridinin), Emiliania huxleyi (19'-hex-fucox), Dunaliella tertiolecta (lutein), and Synechococcus bachllaris (zeaxanthin). Time was normalized by $\mu$, i.e. $t \times \mu$. Values of $R$ and $t \times \mu$ for lutein were divided by a factor of 3 to facilitate presentation of the data. Data were offset on the $R$-axis by multiples of 0.1 units to facilitate presentation

By subjecting cultures to light shifts at the beginning of the labeling experiment, we characterized the ${ }^{14} \mathrm{C}$-labeling kinetics of fucoxanthin in photoadapting Thalassiosira weissflogii. Growth of the light-shifted cultures was unbalanced as evidenced by carbon to fucoxanthin production ratios which differed by a factor of 6 between the high- and lowlight shifted cultures (Table 3). Specific rates of fucoxanthin concentration change were not affected by the light treatments. The turnover rate of fucoxanthin was not significantly different from zero for the high light treatment. Although the turnover rate of fuco-

Table 3. Results of the laboratory light-shift experiments. $\mu_{\text {fucox }}$ was calculated from fucoxanthin concentration changes, the ratio of carbon fixation to fucoxanthin synthesis (C/fucoxprod.) was calculated from the the rates of carbon fixation and fucoxanthin concentration change, and $k_{p}$ and $k_{F}$ were estimated from $P_{\text {fucax }}(t)$ as described in the text. Significance levels for the hypothesis that $k_{\mathrm{p}}$ is different from zero are indicated as given in Table 2

\begin{tabular}{|lccrc|}
\hline & $\begin{array}{l}\mu_{\text {lucox }} \\
\left(\mathrm{d}^{-1}\right)\end{array}$ & $\begin{array}{c}\text { C/fucox-prod. } \\
\left(\mu \mathrm{g} C \mu \mathrm{g}^{-1} \text { fucox }\right)\end{array}$ & $\begin{array}{c}k_{\mathrm{p}} \\
\left(\mathrm{d}^{-1}\right)\end{array}$ & $\begin{array}{c}k_{\mathrm{F}} \\
\left(\mathrm{d}^{-1}\right)\end{array}$ \\
\hline Low light & 1.11 & 51 & $-0.13 \cdots$ & 2.10 \\
Control & 1.27 & 126 & $0.12 \cdots$ & 2.92 \\
High light & 0.96 & 301 & $-0.02^{\mathrm{ns}}$ & 5.97 \\
\hline
\end{tabular}

xanthin was significantly larger than zero for the control and significantly less than zero for the low-light treatments, deviations of these values from zero were still small. Turnover rates of the precursors varied with light treatments, ranging from 2.1 to 6.0 .

\section{Field studies}

\section{Long-term labeling experiments}

A time course labeling experiment lasting $5 \mathrm{~d}$ was performed in the subarctic Pacific. This experiment was designed to test the hypothesis that the F-P model describes the labeling kinetics of pigments in phytoplankton growing under a natural photocycle, as long as rates are integrated over the $24 \mathrm{~h}$ photoperiod. Seawater was incubated at dawn with ${ }^{14} \mathrm{CO}_{2}$ and sampled every $24 \mathrm{~h}$. Whereas concentrations of fucoxanthin increased 15-fold during the experiment, concentration changes of $19^{\prime}$-hex-fucox and $19^{\prime}$-butanoyloxy-fucoxanthin (19'-but-fucox) were small (Fig. 3A). Plots of ' $R$ vs $t$ (Fig. 3B) were similar to those observed in the laboratory for fucoxanthin and 19'-hex-fucox. Labeling patterns predicted by Eq. (1) (solid lines in Fig. 3B) compared well with our measurements.

Specific rates of pigment concentration change, $\left.\left(\ln \mathrm{P}\left[t_{1}\right]-\ln \mathrm{P}\left[t_{0}\right]\right) / \Delta t_{1}\right)$ and specific rates of pigment synthesis (Eq. 1 solved for $\mu$ for measured $P$ and $\Delta t_{i}$ ) were calculated for progressively longer incubation intervals $\left(\Delta t_{1}=t_{1}-t_{0}\right.$, where $t_{l}$ is the sampling time $i_{1}$ and $t_{0}$ is the beginning of the experiment). Specific rates of pigment concentration change were always lower than specific rates of pigment synthesis (Fig. $3 C, D)$, as expected since it is likely that microzooplankton grazing occurred in the incubation bottles (Strom \& Welschmeyer 1991). The specific rate of fucoxanthin synthesis increased from 0.7 to $0.9 \mathrm{~d}^{-1}$ over the course of the experiment; specific rates of $19^{\prime}$-hex-fucox and $19^{\prime}$-but-fucox synthesis were lower by about a factor of 2 to 3 ranging from 0.21 to 0.34 $\mathrm{d}^{-1}$ (Fig. 3C, D). For comparison we included in Fig. 3D specific rates of chl a concentration change and synthesis (Goericke \& Welschmeyer 1993). These rates increased over the $5 \mathrm{~d}$ incubation period from values of 0.0 to $0.3 \mathrm{~d}^{-1}$ and 0.3 to $0.6 \mathrm{~d}^{-1}$, respectively (Fig. 3D). 
Diel labeling patterns

Two $24 \mathrm{~h}$ diel labeling experiments were performed in the subarctic Pacific. Concentrations of peridinin, 19'-hex-fucox and 19'but-fucox did not change over the $24 \mathrm{~h}$ incubation period; however, the concentration of fucoxanthin increased slightly (Figs. $4 \mathrm{~A}$ \& $5 \mathrm{~A})$. The relative specific activity of the carotenoids increased continuously over the entire $24 \mathrm{~h}$ period. Plots of the transformed relative specific activities of the pigments (Figs. 4B \& 5B) closely resembled the labeling patterns observed in the laboratory. The initial curvature of the labeling patterns, which is due to the isotopic disequilibrium between the pool of inorganic carbon and the pigment precursor pool, is particularly evident for these short-term incubations (Figs. $4 \mathrm{~B} \& 5 \mathrm{~B}$ ). These labeling patterns indicate that pigments were synthesized at approximately constant rates throughout the $24 \mathrm{~h}$ incubation period (Figs. 4C \& 5C). Thus, rates of carbon fixation and pigment synthesis were clearly unbalanced in these experiments, since carbon was not fixed in the dark (Goericke \& Welschmeyer 1993)

\section{$P$ vs $I^{14} \mathrm{C}$-labeling experiment}

A $P$ vs $I$ (photosynthesis vs light) experiment was conducted in Chesapeake Bay in September 1984 to study the effects of photoadaptation on patterns of pigment synthesis in the field. Water was sampled from a depth of $2 \mathrm{~m}$ and incubated at light intensities corresponding to $100,44,24,16,8$, and $0.8 \%$ of the surface light intensity $\left(\% I_{0}\right)$. Fucoxanthin

Fig. 3. A $5 \mathrm{~d}$ pigment-labeling experiment performed in the subarctic Pacific in September 1987. Values are plotted against time (d). (A) Concentrations of fucoxanthin, 19' -hex-fucox and 19'-butfucox $\left(\mu \mathrm{g} \mathrm{l}^{-1}\right)$. (B) Transformed relative specific activities $\left({ }^{\circ} R\right)$ of the xanthophylls. Values for 19'but-fucox and fucoxanthin are offset by 0.25 and 0.5 units respectively. Lines represent the predicted time course of $\cdot R$ (Eq. 1) using the mean growth rate determined for the 5 time points and the values for $k_{\mathrm{F}}$ as listed in Table 1. (C) Specific rates of pigment concentration change and specific rates of pigment synthesis for 19'-hex-fucox and $19^{\prime}$-but-fucox. These rates were calculated for $\Delta t=$ $t_{1}-t_{0}$. (D) As in (C), but for fucoxanthin and chl a
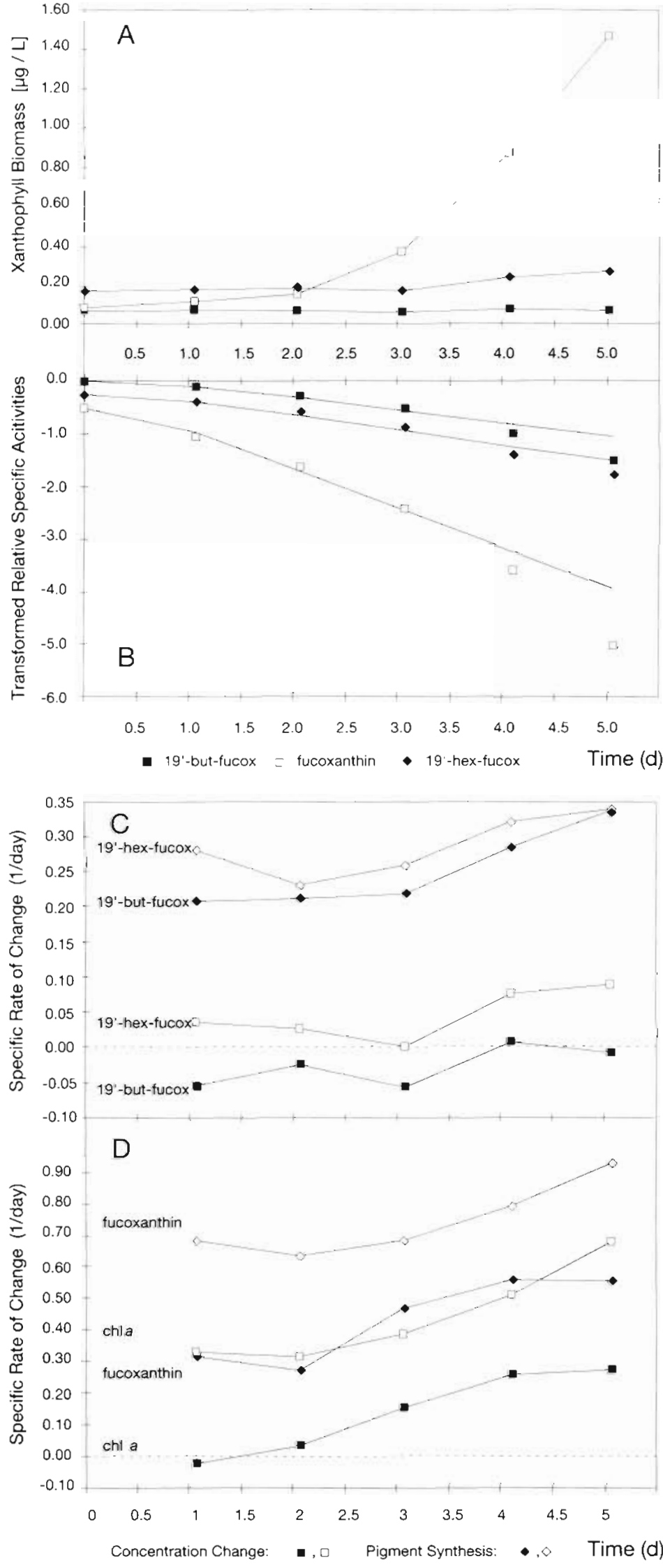


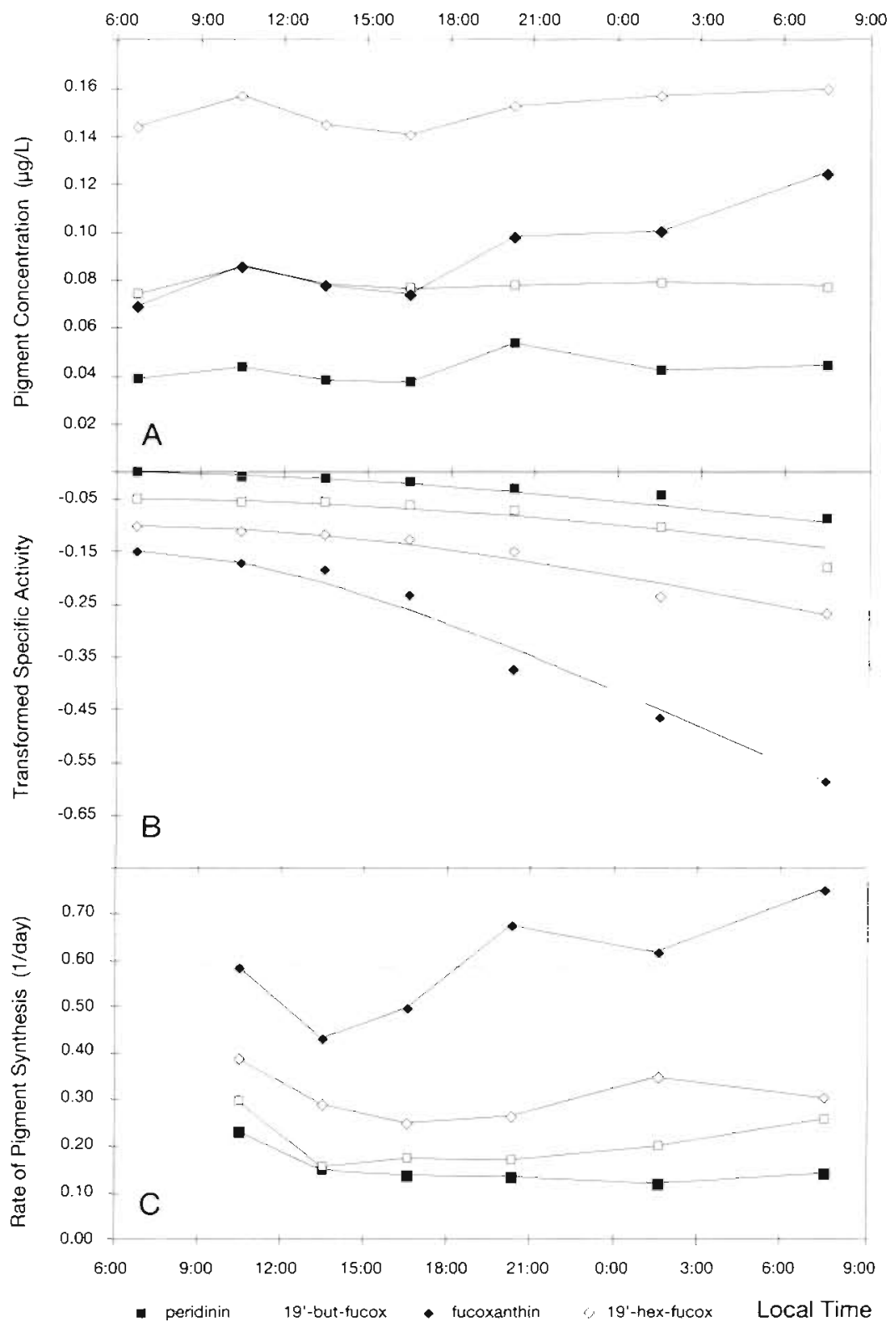

Fig. 4. A 24 h labeling experiment performed in the subarctic Pacific, 21 Sep 1987 Data are calculated and presented as described in Fig. 3. (A) Concen-

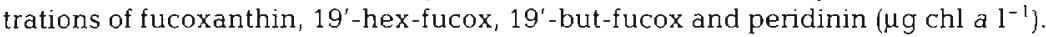
(B) Transformed relative specific activity of the xanthophylls. Solid lines represent a fit of the F-P model to the data. (C) Specific rates of pigment synthesis. Note that rates of peridinin synthesis may include a component due to turnover

and alloxanthin, pigments primarily associated with diatoms and cryptophytes, respectively (Goodwin \& Britton 1988), dominated the carotenoids at the station. Specific rates of pigment concentration change and synthesis were calculated as described above for the subarctic Pacific experiments. These rates were converted to the amount of pigment synthesized (ng pigment $1^{-1}$ ) since the beginning of the experiment. At all light intensities, but $0.8 \% \quad I_{0}$, changes in pigment concentrations were small relative to the amounts of pigment synthesized (Figs. 6 \& 7). Differences between pigment concentration changes and amounts of pigment synthesized were most likely due to grazing. Amounts of fucoxanthin and alloxanthin synthesized at $0.8 \% I_{0}$ were very small. At all other light intensities fucoxanthin was predominantly synthesized during the day and during the early night (Fig. 6B). At night rates of fucoxanthin synthesis were highest at 100,44 and $24 \% I_{0}$. Over the entire incubation period (Fig. $6 \mathrm{~B}, 05: 15 \mathrm{~h})$ about equal amounts of fucoxanthin were synthesized at these 3 highest light levels; amounts of fucoxanthin synthesized declined with irradiance below $24 \% I_{0}$. Concentration changes of alloxanthin were negligible at all light intensities (Fig. 7A). Alloxanthin was primarily synthesized during the first half of the day (Fig. 7B, 14:45 h). Amounts of alloxanthin synthesized during the day were about equal at 100,44 and $24 \% \quad I_{0}$ and declined at the lower light intensities (Fig. 7B). Rates of alloxanthin synthesis were negligible at night.

\section{DISCUSSION}

\section{The carotenoid-labeling method}

The objective of the laboratory experiments was to find an equation that describes ${ }^{14} \mathrm{C}$-labeling kinetics of carotenoids and use such an equation to calculate specific rates of carotenoid synthesis for natural phytoplankton populations. The simple precursorpigment model (Eq. 1) described the ${ }^{14} \mathrm{C}$-labeling data of most carotenoids well. Its free parameters are the specific rates of carotenoid synthesis, $\mu_{\text {carr }}$, the turnover rate of the pigment, $k_{\mathrm{P}}$, and the turnover rate of the pigment's precursor, $k_{\mathrm{F}}$. We used Eq. (1) to calculate taxon-specific growth rates for natural populations of phytoplankton from measured values of $P$ and incubation time $t$. However, results of our experiments suggested that it is only possible to measure the specific rate of pigment synthesis; in cultures this rate corre- 
sponds to the specific rate of pigment concentration change if the pigment does not turn over. Specific rates of pigment concentration change correspond to rates of growth only if growth is balanced. Thus, the following assumptions are made when using the carotenoid-labeling method to determine taxon-specific growth rates in the field:

Assumption 1. The F-P model accurately predicts the ${ }^{14} \mathrm{C}$-labeling patterns of carotenoids in phytoplankton. The labeling time course of most pigments was well described by the F-P model (Fig. 2). An analysis of residuals (data not shown) demonstrated the absence of systematic deviations from the labeling kinetics predicted for fucoxanthin, 19'-hex-fuco, peridinin, lutein, and zeaxanthin. Deviations from labeling patterns as predicted by the F-P model were only observed for $\beta$-carotene in Synechococcus bacillaris (Fig. 1C) and diadinoxanthin in Thalassiosira weissflogii (Goericke \& Welschmeyer 1992b). The labeling kinetics of these 2 pigments were consistent with the presence of at least 2 kinetically distinct pools. These results suggest that it is only possible to use Eq. (1) for the calculation of specific rates of pigment synthesis for those pigments that are present in the algae as single, kinetically distinct pools.

The F-P model was derived assuming that the labeling kinetics of a pigment's immediate precursor, $\cdot F(t)$, is described by ${ }^{*} F=1-\exp \left\{\left(k_{\mathrm{F}}+\mu_{\mathrm{P}}\right) t\right]$; this equation implies that the precursor is directly synthesized from dissolved inorganic carbon (Goericke \& Welschmeyer 1992a). This assumption is clearly wrong since $\alpha$ - or $\beta$-carotene, the precursors of all xanthophylls studied here, are not directly synthesized from dissolved inorganic carbon (Britton 1988). However, the assumption may be adequate for our purpose if the labeling of the precursors of $\alpha$ - or $\beta$-carotene is instantaneous compared to the labeling of the xanthophylls.

To test this assumption we determined the ${ }^{14} \mathrm{C}$-labeling patterns of zeaxanthin and $\beta$-carotene in Synechococcus bacillaris (Fig. 1B, C). If the assumption is correct we must find one $\beta$-carotene pool with a turnover rate similar to the turnover rate of the precur-

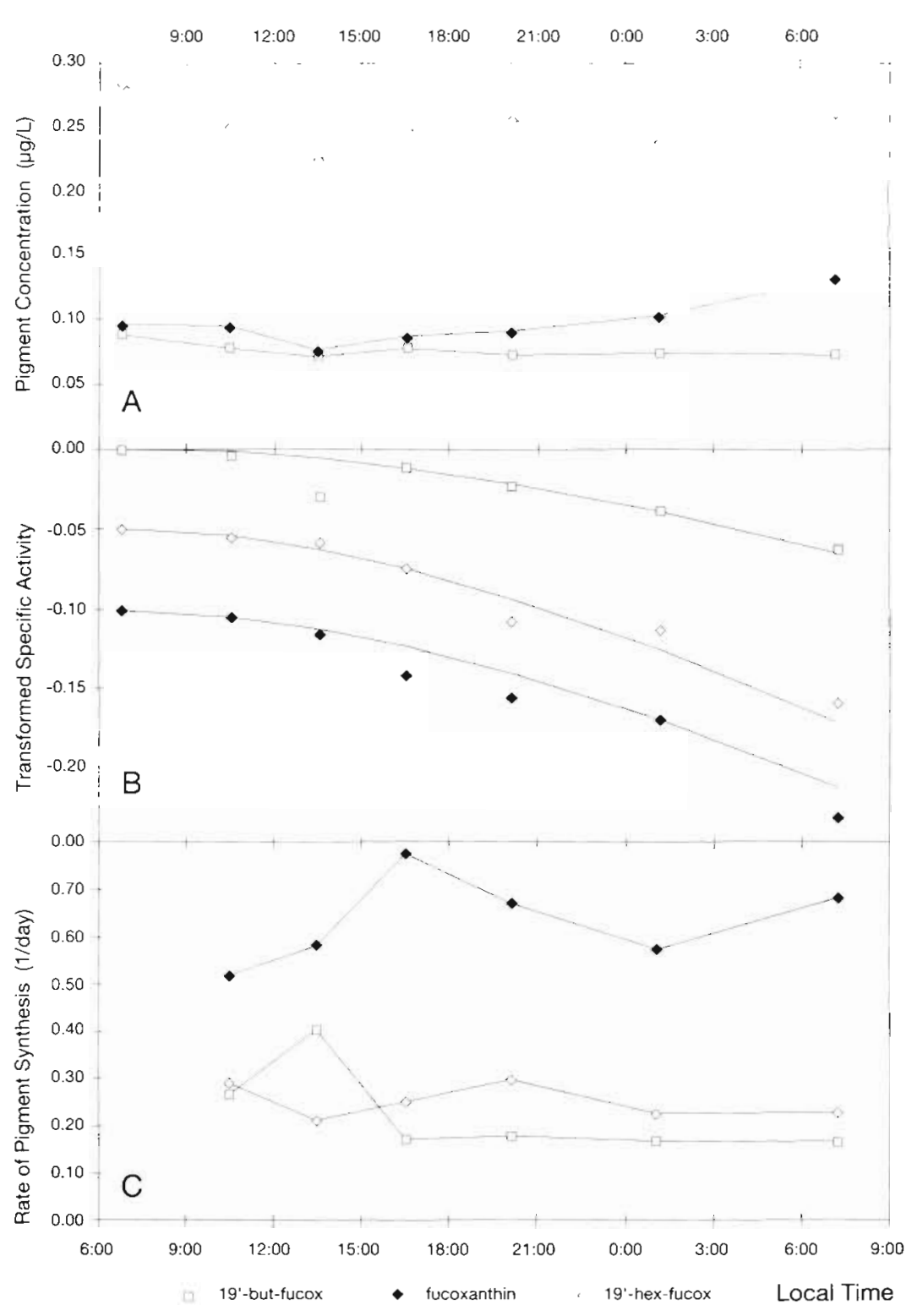

Fig. 5. A $24 \mathrm{~h}$ labeling experiment performed in the subarctic Pacific, 4 Oct 1987. (A) to (C) correspond to Fig. 4 A to $C$. Note that $\cdot R_{\text {fucox }}$ was scaled down by a factor of 2 in Fig. 5B

sor of zeaxanthin. The precursor of this pool of $\beta$ carotene must have a very high turnover rate. Indeed, we detected 2 pools of $\beta$-carotene; the GRN-turnover rate of the small $\beta$-carotene pool, 11, was very similar to the GRN-turnover rate of the zeaxanthin precursor, 16. These results are consistent with the assumption that $\beta$-carotene is a precursor of zeaxanthin. The GRNturnover rate of the precursor of $\beta$-carotene was 371 . Assuming that $S$. bacillaris grows at a rate of $1 \mathrm{~d}^{-1}$ and neglecting isotopic fractionation we expect that the precursor of $\beta$-carotene attains a relative specific activity of 0.99 after $18 \mathrm{~min}$ whereas zeaxanthin requires 


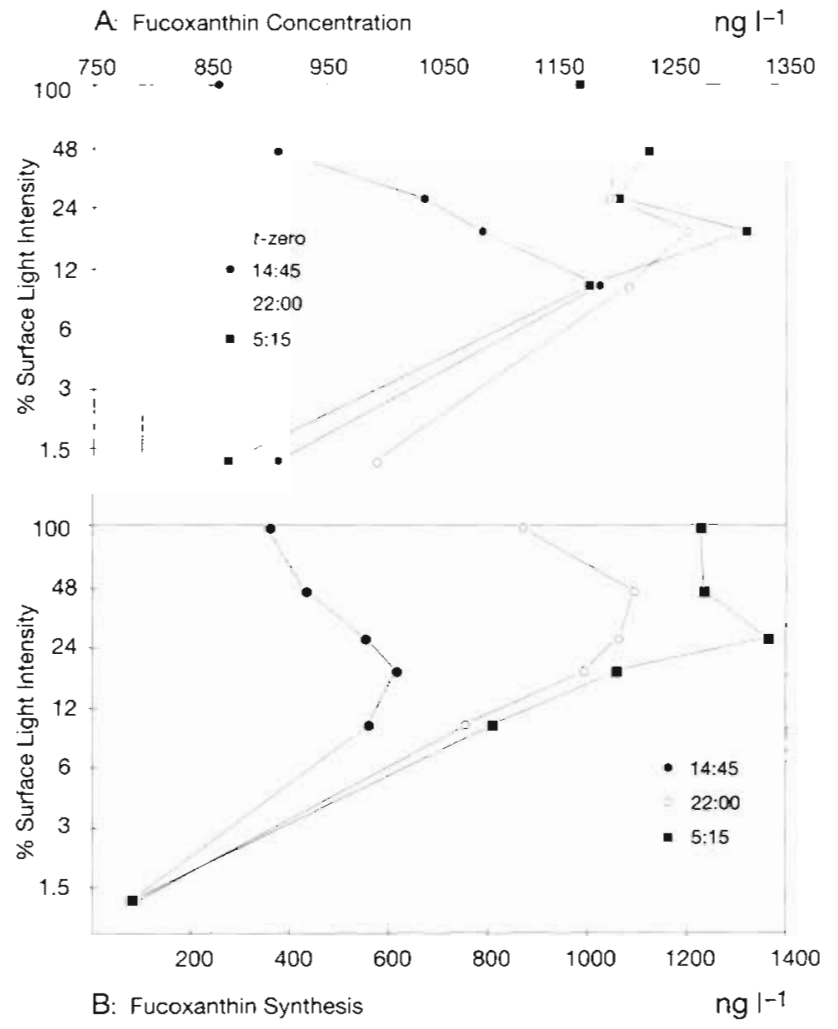

Fig. 6. A $P$ vs $I$ carotenoid-labeling experiment in Chesapeake Bay (2 Oct 1984, Stn 904; see Horrigan et al. 1990 for hydrographic details). The vertical axis represents the light intensity expressed as the percentage of the surface light intensity $\left(\% I_{0}\right)$ at which bottles were incubated. Incubations were started at $t_{0}=09: 45 \mathrm{~h}$ in the morning. Time points were taken at $t_{1}=14: 45, t_{2}=22: 00$ and $t_{3}=05: 15 \mathrm{~h}$ the next morning. (A) Fucoxanthin concentration, ng $\mathrm{l}^{-1}$. The vertical line represents the initial fucoxanthin concentration. (B) Fucoxanthin synthesis $\left(\mu \mathrm{g} \mathrm{l}^{-1}\right)$ during the time interval $\Delta t=t_{i}-t_{0}$ calculated from ${ }^{\prime} P_{\text {fucox }}$ using Eq. (1)

$5 \mathrm{~d}$ to attain this activity. This supports our assumption at least for the case of $S$. bacillaris, that the precursor of $\beta$-carotene is labeled instantaneously when compared to the labeling of zeaxanthin.

Assumption 2. Xanthophylls in marine microalgae do not turn over. The turnover rates of most carotenoids studied by us were not significantly different from zero. These observations were made with microalgae growing in batch cultures under steady state conditions (Table 2) and with cultures of Thalassiosira weissflogii which were photoadapting (Table 3). These results extend our previous observations for chl $a$, chl $b$ and chl $c$ (Goericke 1990, Goericke \& Welschmeyer 1993). So far we have only observed non-zero turnover rates for peridinin in Amphidinium carterae and diadinoxanthin and diatoxanthin in $T$. weissflogii. The turnover of diadinoxanthin and diatoxanthin was discussed previously (Goericke \& Welsch- meyer 1992b); it is likely that these 2 pigments turn over in chromophytes due to their participation in photoprotective mechanisms. At the present time we are not able to suggest a cause for the observed turnover of peridinin in $A$. carterae; however, we can rule out unbalanced growth or other experimental artifacts since the turnover rate of chl a was not significantly different from zero in A. carterae (Goericke \& Welschmeyer 1993). Clearly, more labeling experiments should be performed with dinoflagellates to confirm this result. Thus, it is not possible to use Eq. (1) to determine dinoflagellate or chromophyte growth rates from the labeling of peridinin, diadinoxanthin or diatoxanthin.

Studies of the dynamics of intracellular concentration changes of pigments in microalgae in response to irradiance shifts corroborate these results; cellular concentrations of $\mathrm{chl} a$ in algae adapting to higher light in. tensities decrease due to partial cessation of chl a synthesis and the dilution of cellular chl a due to growth, but not due to degradation, i.e. pigment turnover (e.g. Post et al. 1984). Results of these and our studies are contrary to a previous report of high rates of chlorophyll and carotenoid turnover in Chlorella pyrenoidosa (Grumbach et al. 1978). We have discussed this study previously (Goericke \& Welschmeyer 1992a, b)

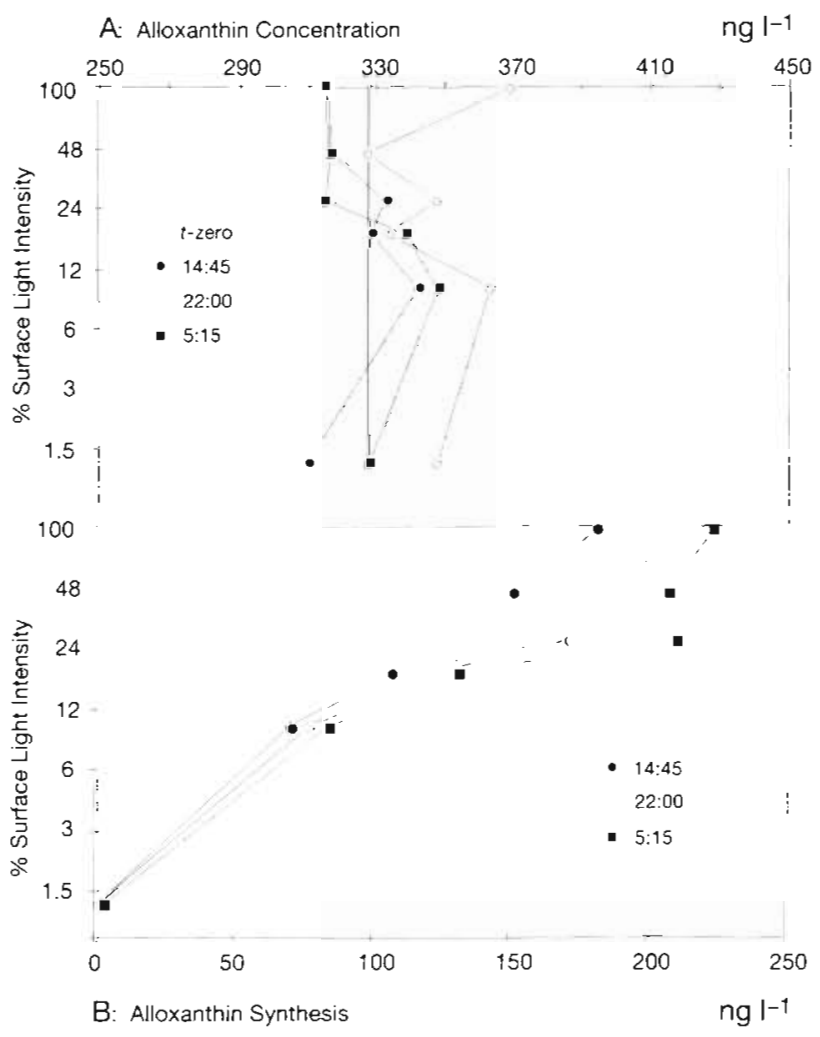

Fig. 7. As in Fig. 6, but for labeling experiments with alloxanthin 
and concluded that these differences may be due to the physiological state of the algal culture that was used.

Assumption 3. For each carotenoid a constant value has to be assumed for the GRN-turnover rate of the carotenoid precursor. We did not observe any systematic variations of GRN-turnover rates of the precursors of chl a or fucoxanthin with growth rate or light intensity in batch cultures of Thalassiosira weissflogii (Goericke \& Welschmeyer 1992a, b). Thus we will assume that GRN-values of $k_{F}$ for individual carotenoids do not vary among species or vary as a function of environmental or physiological parameters. Clearly, the assumption in the form as stated is rather stringent. Its validity must still be established for carotenoids other than fucoxanthin.

The GRN-turnover rates of the precursors of fucoxanthin and 19'-hex-fucox in Phaeodactylum tricornutum and Emiliania huxleyi were 3.8 and 7.6, respectively. These values are not significantly different from 5 , assuming coefficients of variation for these estimates of $30 \%$. Thus, we assumed that the GRN-turnover rates of the precursors of fucoxanthin, 19'-hex-fucox and $19^{\prime}$-but-fucox are identical, and equal to the value of 5 which was previously determined for Thalassiosira weissflogii (Goericke \& Welschmeyer 1992b). The GRN-turnover rates of the precursors of peridinin, lutein and zeaxanthin ranged from 15 to 38 . The GRNturnover rate of the precursor of alloxanthin was not experimentally determined. Alloxanthin $\left(7,8,7^{\prime}, 8^{\prime}\right.$ tetradehydro-, $\beta, \beta$-carotene- $3,3^{\prime}$-diol), the dominant carotenoid in cryptophytes, is an acetylenic carotenoid like diatoxanthin (Goodwin \& Britton 1988). Its biosynthesis may proceed via $\beta$-carotene, cryptoxanthin and crocoxanthin. None of these pigments are present in large amounts in cryptophytes (Hager \& Stransky 1970). Thus, it is likely that the value of the GRNturnover rate of the precursor of alloxanthin is similar to the GRN-turnover rates of the precursors of diatoxanthin, zeaxanthin, or lutein. A value of 20 was thus assumed. Values of the GRN-turnover rates of the carotenoid precursors that were used to calculate growth rates of field population of algae are listed in Table 1

Assumption 4. Rates of growth and rates of pigment synthesis are balanced. We have shown above that it is possible to calculate specific rates of pigment synthesis from rates of ${ }^{14} \mathrm{C}$-incorporation into pigments. However, the conceptual models that we developed (Goericke \& Welschmeyer 1992a, b) suggest that rates of ${ }^{14} \mathrm{C}$-incorporation into carotenoids scale with rates of carotenoid synthesis rather than rates of carbon fixation or cell growth when growth is unbalanced. We tested this hypothesis with a light-shift experiment with the diatom Thalassiosira weissflogii. The effec- tiveness of the light treatments was evidenced by carbon to fucoxanthin production ratios for the high-and low-light-shifted cultures which differed by more than $100 \%$ from the corresponding ratio determined for the control culture (Table 3 ). This experiment corroborated our hypothesis since specific rates of fucoxanthin concentration change and synthesis, as measured using the labeling technique, did not differ by more than $15 \%$.

These results imply that the specific rate of pigment synthesis as determined from the ${ }^{14} \mathrm{C}$-labeling of the pigment corresponds to the growth rate of the phytoplankton only if growth is balanced. Whereas it is likely that phytoplankton growth in dilute batch cultures grown under continuous light is balanced, phytoplankton growth under a natural photocycle may not be balanced at any point of time. However, it is likely that rates of carbon fixation or growth and pigment synthesis are balanced once rates are integrated over the diel photocycle (Eppley 1981).

\section{Testing the method in the field}

The hypothesis that the F-P model predicts carotenoid-labeling patterns of phytoplankton grown under a natural light cycle cannot be conclusively proven in the field, since the true rate of carotenoid synthesis and the true rate of growth cannot be determined by any independent means. However, support for this hypothesis is provided from 2 lines of evidence.

The labeling patterns observed in the subarctic Pacific (Figs. 3B, 4B \& 5B) were very similar to labeling patterns predicted by the F-P model, thus corroborating our hypothesis. The subarctic Pacific labeling experiments also illustrate the importance of correcting labeling data for the time lag due to the isotopic disequilibrium between the pools of inorganic carbon and pigment precursors. This time lag is large for fucoxanthin, 19'-hex-fucox, and 19'-but-fucox. The rate of pigment synthesis for the $3 \mathrm{~h}$ time points would have been severely underestimated had this time lag not been taken into account. The good fit of the F-P model to the 3 and $6 \mathrm{~h}$ time points (Figs. 4B \& 5B) indicates that the assumed values for the GRN-turnover rates $\left(k_{\mathrm{F}}\right)$ of the precursors of the different pigments correspond well to the actual values of these parameters for these natural populations.

We can compare our pigment-based estimates of phytoplankton growth in the subarctic Pacific with concurrent, independent estimates which were based on the dilution technique of Landry \& Hasset (1982). Strom \& Welschmeyer (1991) measured phytoplankton growth rates of $0.54 \mathrm{~d}^{-1}$ for fucoxanthin, $0.17 \bar{d}^{-1}$ for $19^{\prime}$-hex-fucox, and $0.19 \mathrm{~d}^{-1}$ for $19^{\prime}$-but-fucox (Table 4). We measured rates of $0.71 \mathrm{~d}^{-1}$ for fucoxanthin, $0.28 \mathrm{~d}^{-1}$ 
Table 4. A comparison of specific rates of pigment synthesis $\left(d^{-1}\right)$ determined in the subarctic Pacific in September of 1987 : reported by us $\left[24 \mathrm{~h}\right.$ time course experiments, $21 \mathrm{Sep}$ and 2 Oct $_{i} 24 \mathrm{~h}$ time point for the $5 \mathrm{~d}$ labeling experiment for whole seawater (WSW) and for $10 \mu \mathrm{m}$ prescreened seawater (table heading $10 \mu \mathrm{m}$ ) reported in Goericke (1990)] and those reported by Strom \& Weischmeyer (1991), (table heading S \& W '91) who measured growth rates using the dilution technique of Landry $\&$ Hasset (1982). All values are for 24 h incubations only. nd: not determined

\begin{tabular}{|c|c|c|c|c|c|c|}
\hline & 21 Sep & $2 \mathrm{Oct}$ & $\begin{array}{l}25 \text { Sep } \\
\text { WSW }\end{array}$ & $\begin{array}{l}25 \mathrm{Sep} \\
10 \mu \mathrm{m}\end{array}$ & $\begin{array}{c}\text { Average } \\
\bar{\mu} \pm \mathrm{SD}\end{array}$ & $\begin{array}{c}\mathrm{S} \& \mathrm{~W} \cdot 91 \\
\bar{\mu} \pm \mathrm{SD}\end{array}$ \\
\hline Peridinin & 0.14 & 0.19 & nd & nd & $0.17 \pm 0.04$ & $0.24 \pm 0.15(n=4)$ \\
\hline $19^{\prime}$-but-fucox & 0.26 & 0.17 & 0.21 & 0.21 & $0.21 \pm 0.04$ & $0.19 \pm 0.03(n=4)$ \\
\hline Fucox & 0.75 & 0.68 & 0.68 & 0.71 & $0.71 \pm 0.03$ & $0.54 \pm 0.07 \quad(\mathrm{n}=4)$ \\
\hline $19^{\prime}$-hex-fucox & 0.30 & 0.23 & 0.28 & 0.29 & $0.28 \pm 0.03$ & $0.17 \pm 0.03 \quad(n=4)$ \\
\hline Chl a & 0.35 & 0.27 & 0.33 & 0.31 & $0.32 \pm 0.03$ & $0.35 \pm 0.13 \quad(n=4)$ \\
\hline
\end{tabular}

for $19^{\prime}$-hex-fucox and $0.21 \mathrm{~d}^{-1}$ for $19^{\prime}$-but-fucox (Table 4). This close correspondence between rates of growth measured with 2 different methods provides further support for the hypothesis that the F-P model accurately predicts carotenoid labeling patterns of phytoplankton in nature.

\section{Diel labeling patterns}

A variety of physiological processes in microalgae, such as rates of photosynthesis, nutrient uptake, and cell division (Sourina 1974, Chisholm et al. 1986, Waterbury et al. 1986), are affected by the natural photocycle. In phytoplankton cultures grown under a diel photocycle pigments are generally synthesized during the light period; pigment synthesis during the dark period is usually negligible (Eppley et al. 1967, Owens et al. 1980, Post et al. 1984, Kohata \& Watanabe 1989). Redalje (1983) observed balanced rates of carbon fixation and pigment synthesis in a laboratory culture of Mantoniella sp. and in natural phytoplankton populations in the Southern California Bight (USA), whereas we observed an extreme imbalance between rates of pigment synthesis and carbon fixation in the subarctic Pacific (Figs. 4 \& 5). The Chesapeake Bay P vs $I$ experiment high-light treatments displayed diel labeling patterns for fucoxanthin and chl a very similar to those observed in the subarctic Pacific; both pigments were synthesized throughout the incubation period (Fig. 6B) (Goericke \& Welschmeyer 1993). However, the Chesapeake Bay low-light treatments displayed negligible rates of fucoxanthin and chl a synthesis at night. Alloxanthin did not display such light-intensity-dependent patterns of synthesis; it was primarily synthesized during the day at all light intensities (Fig. 7B).

Clearly, complex and potentially interesting physiological phenomena must have been responsible for the observed labeling patterns. However, at the present time it is not possible to interpret these data or even predict the diel labeling patterns of pigments. Nonetheless, it is easy to draw conclusions from these experiments regarding the use of the carotenoid-labeling method in the field: any possible diel labeling pattern may be encountered in the natural environment. Although pigment synthesis in some algal groups, cryptophytes (Fig. 7) or prasinophytes (Redalje 1983), or in some environments, e.g. the Southern California Bight (Redalje 1983), may be directly coupled to rates of carbon fixation, we can not assume this to be the case a priori. Thus, carotenoid-labeling experiments should last $24 \mathrm{~h}$ such that unbalanced growth caused by the diel photoperiod does not bias estimates of growth. Short-term rates of carbon specific growth can only be measured with the carotenoid-labeling method when it is known that instantaneous rates of pigment synthesis and carbon fixation are balanced.

\section{Effects of photoadaptation on patterns of carotenoid synthesis}

The F-P model, the model that we used to describe the ${ }^{14} \mathrm{C}$-labeling kinetics of carotenoids, suggests that ${ }^{14} \mathrm{C}$-labeling rates of pigments scale with rates of pigment synthesis rather than rates of ${ }^{14} \mathrm{C}$-fixation when growth is unbalanced. The laboratory light-shift experiment corroborated this prediction; specific rates of ${ }^{14} \mathrm{C}$-incorporation into pigments and total cell carbon differed by more than a factor of 2 in cultures which were photoadapting. However, we observed that specific rates of pigment synthesis calculated from ${ }^{14} \mathrm{C}$ labeling patterns of pigments corresponded well to specific rates of pigment concentration change. These results suggest that it is not possible to determine rates of phytoplankton growth from rates of ${ }^{14} \mathrm{C}$-incorporation into pigments when phytoplankton phatoadapt; however, it may still be possible to determine specific rates of pigment synthesis from the ${ }^{14} \mathrm{C}$-labeling patterns of carotenoids. 
Photoadaptation in the field can occur whenever phytoplankton experience light shifts, either due to temporal irradiance changes or due to advection of algae across isolumes. Photoadaptation may also occur when deep mixed layers stratify thermally or when phytoplankton sampled from a deep mixed layer are incubated at fixed isolumes. The influence of the latter process on ${ }^{14} \mathrm{C}$-labeling of carotenoids was investigated in Chesapeake Bay. Mixing in the surface layer of the upper reaches of Chesapeake Bay is vigorous due to tidal activity, so that the depth of the euphotic zone often coincides or exceeds the depth of the mixed layer, as was observed during our cruise (Fig. 6c in Horrigan et al. 1990). Phytoplankton collected from any discrete depth in the Chesapeake Bay are not necessarily adapted to the light intensity corresponding to the sampling depth. Since we collected water from 1 depth and incubated it at 6 different light intensities, the light intensity to which the phytoplankton were adapted did not match the light intensity at which phytoplankton were incubated in at least 5 out of the 6 light treatments.

The phytoplankton responded to the light treatments by photoadapting during the experiment; carbon to chlorophyll production ratios for phytoplankton varied by factors of 2 to 10 as a function of light treatment (Goericke \& Welschmeyer 1993). Although it is not possible to calculate carbon to fucoxanthin production ratios for the different light treatments to prove the unbalance between rates of fucoxanthin synthesis and carbon fixation, it is very likely that rates of fucoxanthin synthesis and carbon fixation were similarly unbalanced. This assumption is supported by the similarity of the $P$ vs $I$ labeling patterns for chl a, fucoxanthin, and alloxanthin for the $05: 15 \mathrm{~h}$ time point (Fig. 8B).

The most interesting result of this $P$ vs $I$ experiment was that the diel labeling patterns of fucoxanthin and chl a clearly varied as a function of the light treatment. Relative to the amount of pigment synthesized during the day, less pigment was synthesized at the lower light intensities at night for both pigments (Figs. 6B \& $7 \mathrm{~B})$. During the day, specific rates of chl $a$ and fucoxanthin synthesis were highest at the $16 \%$ light level, whereas rates of alloxanthin synthesis were saturated at the highest light intensities only (Fig. 8A). The shape of the profiles changed due to unequal rates of pigment synthesis at night, such that the shapes of the profiles for all pigments were similar for the 05:15 h time point. It is unlikely that these patterns of diel pigment synthesis are simply a function of the light intensity since it is generally abserved that phytoplankton in cultures synthesize pigments during the day (see above); light-intensity-dependent deviations from this pattern have, to our knowledge, not been observed. It is possible that these light-intensity-modulated pat-

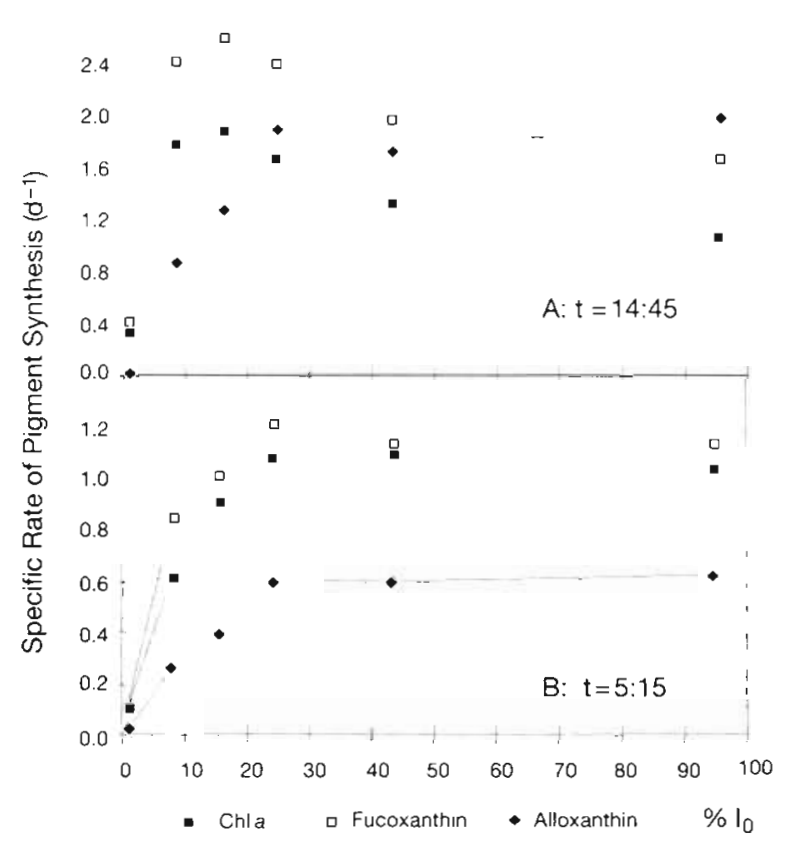

Fig. 8. Specific rates of pigment synthesis plotted as $P$ vs $I$ curves for 2 time points, (A) 14:45 h, and (B) 05:15 h, for the Chesapeake Bay $P$ vs $I$ experiment

terns of diel pigment synthesis represent different strategies of photoadaptation which vary over the diel photocycle depending on the balance between rates of carbon fixation and pigment synthesis.

\section{CONCLUSIONS}

Modifications proposed here for the carotenoidlabeling method are similar to modifications proposed for the chlorophyll-labeling method (Goericke \& Welschmeyer 1993). The most important methodological aspect of the pigment-labeling method is the radiochemical purity of the pigment preparations. Impure pigment preparations can lead to 10 -fold overestimates of pigment activities (cf. Goericke 1992). The most serious limitation of the method is that it is only possible to measure specific rates of pigment synthesis, rather than specific rates of growth. The inference that these rates equal rates of phytoplankton growth has to be justified for each measurement. We have argued that growth is most likely balanced when measurements are averaged over the diel photocycle. Thus incubations should last $24 \mathrm{~h}$, dawn to dawn. However, even measurements integrated over $24 \mathrm{~h}$ are not balanced when phytoplankton photoadapt. Thus, care must be taken to avoid perturbations of the natural light field; in situ incubations are preferable. The results of the Chesapeake Bay light-perturbation ex- 
periment suggest that phytoplankton growth may be unbalanced when the phytoplankton is sampled from a deep mixed layer and incubated at fixed irradiances. It may be preferable to report an average mixed-layer growth rate for those conditions rather than individual growth rates for incubations at fixed isolumes.

The field experiments presented here not only demonstrate physiological principles but also the utility of the carotenoid-labeling method to elucidate ecological processes. In the subarctic Pacific we were able to differentiate between fast growing diatoms (fucoxanthin) and slower growing prymnesiophytes (19'hex-fucox) and chrysophytes (19'-but-fucox) (Figs. 3 to 5). We were able to show that, although the composition of the phytoplankton community changed over the course of the $5 \mathrm{~d}$ incubation (Fig. 3A), growth rates of the components of the community remained almost constant (Fig. 3C, D). This experiment demonstrated that community structure and community growth rates can change in incubation bottles even if the physiological characteristics of the individual populations remain constant. The observed changes of community structure were most likely mediated by the grazer community (see also the discussion of these experiments in the review by Welschmeyer et al. 1991). In Chesapeake Bay, we observed different diel labeling patterns and possibly different $P$ vs $I$ responses for the different groups of algae.

These experiments clearly show the potential of the carotenoid-labeling method to investigate the physiological ecology of phytoplankton and the potential to delineate physiologically distinct groups of algae using taxon-specific carotenoids. The method can be a particularly useful tool in conjunction with perturbation experiments, e.g. light shifts, nutrient additions, and grazer manipulations, to monitor the physiological response of different phytoplankton populations to experimental perturbations. Carotenoid-labeling experiments may contribute to our understanding of the role of the individual phytoplankton population in marine ecosystems

Acknowledgements. We are grateful to Jim McCarthy, Sarah Horrigan and the SUPER group for support during the field studies. We acknowledge S. Chisholm, J. McCarthy, C. Cavanough, C. Arnosti, G. Kleppel and W. Gieskes for helpful comments on the manuscript. This research was supported by NSF grants OCE-8309755 and OCE-8613943 to N.A.W. Preparation and publicaiton of the manuscript was supported by NSF grant OCE9101384 to D. Repeta and R.G

\section{LITERATURE CITED}

Britton, G. (1988). Biosynthesis of carotenoids. In: Goodwin, T. W. (eds.) Plant pigments. Academic Press, London, p. $133-182$
Chisholm, S. W., Armbrust, W. V., Olson, R. J. (1986). The individual cell in phytoplankton ecology: cell cycles and applications of flow cytometry. In: Platt, T., Li, W. K. W. (eds.) Photosynthetic phytoplankton. Dept Fisheries and Oceans, Ottawa, p. 287-309

Davies, R. H. (1976). Carotenoids. In: Goodwin, T. W. (ed.) Chemistry and biochemistry of plant pigments. Academic Press, London, p. 38-165

Douglas, D. J. (1984). Microautoradiography-based enumeration of photosynthetic picoplankton with estimates of carbon-specific growth rates. Mar. Ecol. Prog. Ser. 14: $223-228$

Eppley, R. W. (1981). Relations between nutrient assimilation and growth in phytoplankton with a brief review of estimates of growth rates in the ocean. Can. Bull. Fish. Aquat. Sci. 210: 251-263

Eppley, R. W., Holmes, R. W., Paasche, E. (1967). Periodicity in cell division and physiological behaviour of Ditylum brightwellii, a marine planktonic diatom, during growth in light-dark cycles. Arch. Mikrobiol. 56: 305-323

Fiksdahl, A., Mortensen, J. T., Liaaen-Jensen, S. (1978) High-pressure liquid chromatography of carotenoids J. Chromatography $157: 111-117$

Gieskes, W. W. C., Kraay, G. W. (1989). Estimating the carbon-specific growth rate of the major algal species groups in eastern Indonesian waters by ${ }^{14} \mathrm{C}$ labeling of taxonspecific carotenoids. Deep Sea Res. 36: 1127-1139

Goericke, R. (1990). Pigments as ecological tracers for the study of the abundance and growth of marine phytoplankton. Ph.D. thesis, Harvard University, Cambridge

Goericke, R. (1992). The chlorophyll-labeling method: the radiochemical purity of chl $a$. A response to Jespersen et al., 1992, J. Plankton Res. J. Plankton Res. 14: 1781-1785

Goericke, R., Welschmeyer, N. A. (1992a). Pigment turnover in the diatom Thalassiosira weissflogii. I. Labeling kinetics of chlorophyll a with ${ }^{14} \mathrm{CO}_{2}$. J. Phycol. 28: 498-507

Goericke, R., Welschmeyer, N. A. (1992b). Pigment turnover in the diatom Thalassiosira weissflogii. II. Labeling kinetics of carotenoids with ${ }^{14} \mathrm{CO}_{2}$. J. Phycol. 28: 507-517

Goericke, R., Welschmeyer, N. A.. (1993). The chlorophylllabeling method: measuring specific rates of chlorophyll a synthesis in cultures and in the open ocean. Limnol. Oceanogr. 38: 80-95

Goodwin, T W., Britton, G. (1988), Distribution and analysis of carotenoids. In: Goodwin, T. W. (ed.) Plant pigments. Academic Press, London, p. 61-132

Grumbach, K. H., Lichtenthaler, H. K., Erismann, K. H. (1978). Incorporation of ${ }^{14} \mathrm{CO}_{2}$ in photosynthetic pigments of Chlorella pyrenoidosa. Planta (Berl.) 140: 37-43

Hager, A., Stransky, H. (1970). Das Carotinoidmuster und die Verbreitung des lichtinduzierten Xanthophyll Cyclus in verschiedenen Algen Klassen: V. Einzelne Vertreter der Cryptophyceae, Euglenophyceae, Bacillariophyceae, Cryptophyceae und Phaeophyceae. Arch. Mikrobiol. 73: $77-89$

Horrigan, S. G., Montoya, J. P., Nevins, J. L., McCarthy, J. J., Ducklow, H. W., Goericke, R., Malone, T. (1990). Nitrogenous nutrient transformations in the spring and fall in the Chesapeake Bay. Estuar, coast. Shelf Sci. 30: $369-391$

Jeffrey, S. W. (1980). Algal pigment systems. In: Falkowski, P. G. (ed.) Primary productivity in the sea. Plenum, New York, p. 33-58

Jensen, A. (1978). Chlorophylls and carotenoids. In: Helleburst, J. A., Craigie, J. S. (eds.) Handbook of phycological methods: physiological and biochemical methods Cambridge Univ. Press, Cambridge, p. 59-70 
Kohata, K., Watanabe, M. (1989). Diel changes in the composition of photosynthetic pigments and cellular carbon and nitrogen in Pyramimonas parkeae (Prasinophyceae). J. Phycol. 25: 377-385

Landry, M. R. Hasset, R. P. (1982). Estimating the grazing impact of marine microzooplankton. Mar. Biol. 67: $283-241$

Liaaen-Jensen, S. (1985). Carotenoids of lower plants - recent progress. Pure appl. Chem. 57: 649-658

Maron, M. J. (1982). Numerical analysis. Macmillan, New York

McDuff, R. E., Chisholm, S. W. (1982). The calculation of in situ growth rates of phytoplankton populations from fractions of cells undergoing mitosis: a clarification. Limnol. Oceanogr. 27: 783-788

Owens, T. G., Falkowski, P. G., Whitledge, T. E. (1980). Diel periodicity in cellular chlorophyll content in marine diatoms. Mar. Biol. 59: 71-77

Pennington, F. C., Haxo, F. T., Borch, G., Liaaen-Jensen, S. (1985). Carotenoids of Cryptophyceae. Biochem. Syst. Ecol. 13: 215-219

Post, A. F., Dubinsky, Z., Wyman, K., Falkowski, P. G. (1984). Kinetics of light-intensity adaptation in a marine planktonic diatom. Mar. Biol. 83: 231-238

Redalje, D. G. (1983). Phytoplankton carbon biomass and

This article was presented by D. A. Caron, Woods Hole, Massachusetts, USA specific growth rates determined with the labeled chlorophyll a technique Mar. Ecol. Prog. Ser. 11:217-225

Redalje, D. G., Laws, E. (1981). A new method for estimating phytoplankton growth rates and carbon biomass. Mar. Biol. 63: 73-79

Roberts, D. W. A. (1969). The quantitation of pheophorbide $a$, phyropheophorbide $a$, and their esters. Can. J. Biochem. 47: $721-724$

Shuter, B. (1979). A model of physiological adaptation in unicellular algae. J. theor. Biol. 78: 519-552

Sourina, A. (1974). Circadian periodicities in natural populations of marine phytoplankton. Adv. mar. Biol. 12: 325-389

Strom, S. L., Welschmeyer, N. A. (1991). Pigment-specific rates of phytoplankton growth and microzooplankton grazing in the open subarctic Pacific Ocean. Limnol. Oceanogr. 36: 50-63

Waterbury, J. B., Watson, S. W., Valois, F. W., Franks, D. G. (1986). Biological and ecological characterisation of the marine unicellular cyanobacterium Synechococcus. In: Platt, T., Li , W. K. W. (eds.) Photosynthetic picoplankton. Dept Fisheries and Oceans, Ottawa, p. 71-120

Welschmeyer, N. A., Goericke, R., Strom, S. L., Peterson, W. (1991). Phytoplankton growth and herbivory in the subarctic Pacific: a chemotaxonomic analysis. Limnol. Oceanogr. 36: 1631-1649

Manuscript first received: December 23, 1991

Revised version accepted: April 21, 1993 